\title{
Distribución del gasto farmacéutico en medicación antirretoviral
}

\author{
C. TORNERO ESTEBANEZ, A. CUENCA SORIA', A. SANTAMARÍA MARTÍN, \\ E. GIL TOMÁS, E. SOLER COMPANY' ${ }^{1}$, S. RULL SEGURA
}

Servicio de Medicina Interna $y^{\prime}$ Farmacia. Hospital Francisco de Borja. Gandía, Valencia

\section{RESUMEN}

La eficacia de la terapia antirretroviral de gran actividad (TARGA) supone un gasto farmacéutico elevado. La falta de adherencia hace que no todos los pacientes se beneficien de ella y algunos enfermos se encuentran en fases no avanzadas de la enfermedad donde podría no ser necesaria. Nos proponemos valorar cómo se distribuyen los costos de la TARGA.

Material y métodos: Se analizaron 199 pacientes adultos con infección por VIH que recibieron TARGA durante al menos una vez en el año 2001. Con la historia farmacológica de recogida de medicación se obtuvo el costo de la TARGA en todo el grupo, así como en los pacientes que abandonan la medicación (grupo A), en los que no controlan la carga viral (grupo B), y en los que parten o alcanzan CD4+ > $500 \mathrm{cs} / \mathrm{mm} 3$ (Grupos $\mathrm{C} 1$ y $\mathrm{C} 2$ respectivamente).

Resultados: EL gasto global anual de esta medicación, descontando las devoluciones fue de $961.720 \quad(21,3 \%$ del gasto farmacológico total del hospital) del que correspondía al grupo A el 6,3\%, al grupo B el $14,26 \%$ y a los subgrupos $\mathrm{C} 1$ y C2 el 12,1 y el $8,1 \%$ respectivamente.

Comentarios: En nuestro centro casi el $40 \%$ del elevado gasto económico en antirretrovirales se distribuye entre pacientes que no lo cumplen, que no alcanzan la supresión de la carga viral o que, por su situación inmunológica, podrían no necesitarlo.

PALABRAS CLAVE: Terapia antirretroviral. Gasto farmacéutico.
COST DISTRIBUTION OF HIGHLY ACTIVE ANTIRETROVIRAL THERAPY

\begin{abstract}
The efficacy of highly active antiretroviral therapy (HAART) implies a high pharmaceutical cost. Deficient patient adhesion to therapy means that not all individuals benefit from treatment, while some patients are in non-advanced stages of the disease where such management may not be necessary. A study is made of the cost distribution of HAART.

Material and methods: An analysis was made of 199 HIV-infected adults subjected to HAART at least once during 2001. The medication history was used to calculate the cost of HAART in the global group, in the patients who abandoned treatment (group A), in the group in which viral load was not controlled (group B), and in the patients starting with or reaching a CD4+ count $>500$ cells $/ \mathrm{mm} 3$ (groups $C 1$ and $C 2$, respectively).

Results: The global annual cost of HAART (discounting returned medication) was $961,720 \quad$ (21.3\% of the global hospital pharmacological expenditure), group A accounting for 6.3\%, group B $14.26 \%$, and subgroups $C 1$ and $C 2$ for 12.1 and $8.1 \%$, respectively.

Comments: In our center, almost $40 \%$ of antiretroviral drug expenditure may be considered of little use.
\end{abstract}

KEY WORDS: Antiretroviral therapy. Drugs cost.

Tornero Estebanez C, Cuenca Soria A, Santamaría Martín A, Gil Tomás E, Soler Company E, Rull Segura S. Distribución del gasto farmacéutico en medicación antirretoviral. An Med Interna (Madrid) 2004; 21: 269-271.

\section{INTRODUCCIÓN}

La terapia antirretroviral de gran actividad (TARGA) ha demostrado su eficacia en el control de la infección por el VIH reduciendo significativamente las infecciones oportunistas y mejorando la supervivencia de estos pacientes (1). La TARGA supone una proporción considerable del gasto farmacéutico hospitalario, tanto por sus elevados costos de adquisición (2) como por el número de pacientes que en nuestro país están infectados por el VIH.

Pero no todos los pacientes a los que se pauta TARGA se benefician de ella. Son frecuentes las intolerancias medicamentosas, la falta de eficacia por resistencias o incumplimiento y los abandonos del tratamiento, que suponen un considerable gasto económico.

Se podría también considerar, dentro de estos costos menos efectivos, las pautas terapéuticas que se iniciaron siguiendo recomendaciones más agresivas que las actuales, y aquellos pacientes que, tras la reconstitución inmune, podrían en ese momento no requerir antirretovirales.

Nos proponemos valorar como se distribuye el gasto económico de la TARGA en nuestros pacientes con infección por VIH a lo largo de un año.

Trabajo aceptado: 13 de enero de 2004

Correspondencia: C. Tornero. Servidio de Medicina Interna. Hospital Francisco de Borja. Paseo Germanías, 71. 46700 Gandía (Valencia). e-mail: torneo_car@gva.es. 


\section{MATERIAL Y MÉTODOS}

Se analizaron los pacientes adultos con infección por VIH en seguimiento en el Servicio de Medicina Interna del Hospital de Gandía que recibieron TARGA durante al menos una vez en el año 2001. Se excluyeron los tratamientos pautados post-exposición parenteral o sexual y los incluidos en ensayos clínicos.

Con la historia farmacológica de recogida de medicación (programa informático Farmasys) se obtuvo el costo global de la TARGA de todos los pacientes y de los subgrupos como se definen después, descontándose las devoluciones de medicación registradas.

Sobre la base de los seguimientos en consultas externas se clasificaron los pacientes en 3 grupos:

Grupo A: Abandonos de medicación: Aquellos que recogieron medicación una o varias veces pero que no vuelven a las revisiones. Se comprobó tambien los seguimientos de estos enfermos en nuestro centro en los 18 meses siguientes para descartar aquellos que pudieran haberse trasladado a otro centro manteniendo el tratamiento.

Grupo B: Tratamientos no efectivos: Pacientes que pese a recoger la medicación de forma regular, no mantienen cargas virales indetectables o casi indetectables ( $<1.000$ copias/ $\mathrm{mm}^{3}$ ) en más de dos determinaciones consecutivas a lo largo del año. Basándose en la historia clínica y de recogida de medicación se atribuyo este fracaso a dos subgrupos no excluyentes:

B1. Objetivación de resistencias genotípicas

B2. Incumplimiento terapéutico basado en el reconocimiento por el propio paciente, o en la recogida irregular de la medicación durante el periodo de estudio con desfase mayor del $10 \%$.

Grupo C. Pacientes que mantienen con TARGA cargas virales indetectables o casi indetectables $\left(<1.000 \mathrm{cop} / \mathrm{mm}^{3}\right)$. Se incluyen aquí aquellos con "blips", definidos como determinaciones de $\mathrm{cv}<10.000$ copias que no se repite en controles posteriores sin que sea necesario modificar la terapia antirretroviral. También consideramos aquí aquellos que pese a requerir cambios de medicación por falta de eficacia o intolerancia, alcanzan cargas virales indetectables. A su vez se distinguen dos subgrupos de pacientes.

C1) Aquellos que iniciaron el tratamiento con cifras de CD4 $>500 \mathrm{cs} / \mathrm{mm}^{3}$ y que no estaría justificado con las recomendaciones actuales (3).

C2) Pacientes que, sin pertenecer al grupo $\mathrm{C} 1$, mantiene en la mayoría de las determinaciones realizadas durante el año, CD4 por encima de $500 \mathrm{cs} / \mathrm{mm}^{3}$.

\section{RESULTADOS}

En al año 2001 se distribuyó TARGA a 208 pacientes no incluidos en ensayos clínicos. Se excluyeron 5 niños, 3 tratamientos post exposición y un caso cuya historia clínica estaba incompleta. 199 se consideraron útiles para su análisis.

El $63 \%$ era varones. Por grupos de riesgo correspondía $62 \%$ a UDVP, $26 \%$ heterosexuales, $5 \%$ homosexuales y $6 \%$ no especificado.

El gasto global anual de esta medicación, descontando las devoluciones fue de $961.720 €(21,3 \%$ del gasto farmacológico total del hospital) que se distribuyen en los subgrupos A y $\mathrm{B}$ descritos como se recoge en la tabla I. Se dispone de segui-
TABLA I

\begin{tabular}{lccc}
$\begin{array}{c}\text { DISTRIBUCIÓN DEL GASTO FARMACÉUTICO DE LA TERAPIA } \\
\text { ANTIRRETROVIRAL A LO LARGO DE UN AÑO }\end{array}$ \\
$\begin{array}{cccc}\text { Número } \\
\text { pacientes }\end{array}$ & $\begin{array}{c}\text { Costo total (€) } \\
\text { Porcentaje } \\
\text { del global (\%) }\end{array}$ \\
\hline $\begin{array}{l}\text { Grupo A } \\
\text { (Abandonos) }\end{array}$ & $n=34$ & 60771 & 6,31 \\
$\begin{array}{l}\text { Grupo B } \\
\text { (Tratamiento no } \\
\text { efectivo) }\end{array}$ & $\begin{array}{c}\text { Total } n=28 \\
\text { B1 (Resistencias) } \\
n=11\end{array}$ & 137144 & 14,26 \\
& $\begin{array}{c}n \\
\text { B2 (falta de } \\
\text { adherencia) } n=19\end{array}$ & 77334 & 8,04 \\
\hline
\end{tabular}

mientos porteriores al abandono en 25 pacientes del grupo A por lo que no podría excluirse que los restantes hubieran cambiado de domicilio y fueran seguidos en otro centro.

Por otra parte de los 137 pacientes que se incluyeron en el grupo $\mathrm{C}$, 21 se clasificaron como $\mathrm{C} 1$ (pacientes que iniciaron tratamiento con CD4 $>500 \mathrm{cs} / \mathrm{mm}^{3}$ ) y 14 como C2 (aquellos que alcanzan CD4 $>500 \mathrm{cs} / \mathrm{mm}^{3}$ ), lo que suponía un 12,1 y $8,1 \%$ respectivamente del costo total en medicación antirretroviral.

\section{DISCUSIÓN}

Los beneficios de la TARGA son indudables, como lo son los elevados gastos que generan en la farmacia hospitalaria suponiendo en nuestro centro el $21,5 \%$ del total.

El especial entorno sociocultural y la coexistencia con toxicomanías de muchos pacientes con infección por $\mathrm{VIH}$ dificultan los seguimientos y su correcta adherencia, lo que compromete la eficacia y genera resistencias.

Los pacientes que tras recoger medicación no vuelven a consulta sin devolver la no utilizada, suponen un gasto económico completamente inútil, que en nuestra serie alcanzó el 6,3\% del total (mas de 60.000 Euros en un año) lo que resulta especialmente indecente en un mundo donde la mayoría de los infectados por el VIH no tienen posibilidad de acceder a la TARGA. La dispensación de cantidades reducidas y reutilizables de medicación especialmente en pacientes con historia previa de abandonos podría reducir estos costos, y nos llevo en nuestro centro a reducir esta primera dispensación de 4 a 2 semanas.

Otro grupo a considerar son aquellos en los que no se consigue el objetivo terapéutico de supresión de la carga viral, por mala adherencia, o por la existencia de resistencias, que en muchos casos son consecuencia de una mala cumplimentación previa. Resulta destacable que en ocasiones no se objetivan resistencias genotípicas aunque no esté suprima la viremia, lo que en muchos casos podría reflejar un bajo nivel de cumplimiento pese a acudir a revisiones y recoger la medicación de manera regular. Estos pacientes de carga viral persistentemente no suprimida suponen en nuestra serie otro 14,26 $\%$ del gasto económico en antirretrovirales. El abordaje de estrategias para evaluar y mejorar la adherencia, y la adecuación de las formas de dispensación de la medicación (4-7) podría reducir este porcentaje de gasto económico poco eficaz , además de contribuir a evitar la generación de cepas resistentes trasmisibles. 
En los pacientes cuya TARGA es efectiva en controlar la carga viral, hemos destacado también dos grupos donde podría discutirse su retirada temporal dado el bajo riesgo de progresión a corto plazo, la imposibilidad de erradicación del VIH, la toxicidad a largo plazo y los costos económicos. Son aquellos que iniciaron tratamiento y se mantienen en él por la inercia histórica de indicaciones más agresivas que las actuales (3), y también los que se encuentran con cifras persistentemente elevadas de CD4 tras la reconstitución inmunologica $(8,9)$. En nuestra serie, tomando una cifra muy conservadora de $500 \mathrm{cs} / \mathrm{mm}^{3}$, suponen unos porcentajes del 12,1 y $8,1 \%$ de posible reducción de la factura farmacéutica del TARGA, que podría ampliarse considerablemente cuanto más nos aproximemos a los límites cada vez más promulga-

\section{Bibliografía}

1. Palella FJ, Delaney KM, Moorman AS, Loveless MO, Fuhrer J, Satten GA, et al. Declining Morbidity and mortality among Patients with Advanced Human Immunodeficiency Virus Infection. N Eng J Med 1998; 338: 853-860.

2. Sanz A. Coste de los fármacos antirrertrovirales. Offarm 2003; 22: 130-134.

3. Dybul, M, Faucy AS, Barlett JG, Kaplan JE, Pau AK. Guidelines for using antiretroviral agents among HIV-infected adults and adolescents. Ann Intern Med 2002; 137: 381-433.

4. González M. Antón R, Borras J, Ortiz de Urbina V. Inadecuada presentación de las formas farmacéuticas de dosificación de la terapia antirretroviral. Med Clin (Barc) 1999; 113: 559-560.

5. Cabeza J, García MA, Zamora MA, Díez LF. Adecuación de las formas farmacéuticas de dosificación de la terapia antirretroviral. Med Clin (Barc) 2000; 114: 356-357. das de CD4 entre 200 y 350 cs $/ \mathrm{mm}^{3}$ como indicación de terapia antirretroviral. Consideraciones más profundas que el puro ahorro farmaceútico deberían realizarse antes de promulgar la suspensión de la TARGA en estos pacientes, por lo que los datos aquí reflejados sólo tienen un carácter descriptivo.

En resumen en nuestro centro solo un $60 \%$ del elevado gasto económico en antirretrovirales se administra a pacientes en los que la indicación es incuestionable y alcanzan el objetivo terapéutico de suprimir la carga viral.

El entusiasmo inicial por los indudables beneficios de la TARGA que sin duda resultan costo-efectivos (10), debe dejar paso a la reflexión sobre los medios para racionalizar el coste, también en el tratamiento antirretroviral.
6. Puigventós F, Riera M, Delibes C, Penaranda M, De la Fuente L, Boronat A. Estudios de adherencia a los fármacos antirretrovirales. Una revisión sistemática. Med Clin (Barc) 2002; 119: 130-137.

7. Riera M, De de Fuente L, Castanyer B, Puigventós F, Villalonga C, Ribas MA, et al. Adherencia a los fármacos anirretrovirales medida por la concentracion de fármacos y el recuento de comprimidos. Variables relacionadas con una mala adherencia. Med Clin (Barc) 2002; 119: 286-292.

8. Jacobs B, Aoulafia D. Chronic HIV infection. Ann Intern Med 2003; 138: 437-438.

9. Ruiz L, Gómez L, Domingo P, Romeu J, Tambussi G, Martínez-Picado $\mathrm{J}$ et al. A multicenter Randomized controlled clinical trial of continuous vs intermittent HAART guided by CD4 T-cell Counts and plasma HIV1 RNA levels. 10th CROI, Boston, Febrero 2003. Abstract 65.

10. Steinbrook R. Providing antiretroviral therapy for HIV infection. N Eng J Med 2001; 344: 844-845. 